\title{
Retroperitoneal Cystic Lymphangioma: A Case Report of Spontaneous Regression and Review of Literature
} \author{
Guillaume Levard ${ }^{2}$ and Jiad N Mcheik ${ }^{2}$ \\ ${ }^{1}$ Department of Urology, Poitiers University Hospital, France \\ ${ }^{2}$ Department of Pediatric Surgery, Poitiers University Hospital, France
}

Stessy Kutchukian ${ }^{*}$, Diana Potop ${ }^{2}$, Khalil Chalhoub ${ }^{1}$,Emmanuelle Verdier ${ }^{2}$, Marie Auger ${ }^{2}$,

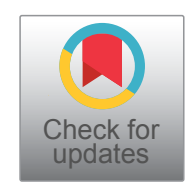

\begin{abstract}
Background: Cystic lymphangioma $(\mathrm{CL})$ is a rare and benign tumor derived from the lymphatic system that can have different localisations. This tumor consists of several numbers of cysts that have cavity-like appearance. During childhood, cystic lymphangiomas are as common in boys as in girls (sex ratio 1:1). This lesion can have a sudden onset, a rapid growth or stability and can even regress spontaneously. It has a variable clinical presentation and sometimes can be asymptomatic. For this rare malformation the final diagnosis is a histological confirmation. Case presentation: We present the case of a 7-year-old girl that was diagnosed before birth with a retroperitoneal CL by ultrasound and Magnetic Resonance Imaging (MRI) with a left lumbar subcutaneous extension associated with a cutaneous lymphangioma. This retroperitoneal lesion remained asymptomatic, non visible clinically and it had a spontaneous resolution without any treatment; however, the cutaneous lesion kept evol-ving.
\end{abstract}

Conclusion: The diagnosis of $\mathrm{CL}$ can be made by imaging during the prenatal period and sometimes during adulthood. The usual treatment for asymptomatic $\mathrm{CL}$ is regular clinical and radiological monitoring. However for symptomatic lesions the first line treatment is surgery. In our case, surgical treatment was performed only for the skin lesions.

\section{Abbreviations}

CL: Cystic lymphangioma; MRI: Magnetic Resonance Imaging; BMI: Body Mass Index; wGA: Weeks Gestational Age; US: Ultrasound; CT: Computed Tomographic

\section{Introduction}

Because of the latest advances in imaging nowadays, it is possible to have a very precise prenatal diagnosis. Cystic lymphangiomas are uncommon tumors, with different localisations. $75 \%$ are located in the cervical region, $20 \%$ in the axillary region and $5 \%$ in other sites including the retroperitoneum $[1,2]$. When compared to the mesentery, the retroperitoneum localisation can be variable according to the cohort studies, with an equal or superior incidence $[3,4] .50$ to $60 \%$ of lesions appear during the first year of life and 80 $90 \%$ are diagnosed in the second year, making them a rare adult entity. Cystic lymphangiomas are thought to develop from parts of the sacs sequestered from primary lymphatic channels during em-bryonic life. There are different theories concerning the pathogenesis of these lesions. The most probable cause in our case is a localized lymphatic stasis due to a congenital obstruction of the regional lymphatic drainage. When a lymphangioma grows, the increase in size is due to a partial or complete lack of communication with the adjacent lymphatic ducts. This leads to multilocular cysts which can be variable in size. They are thin-walled structures and often communicate with each other. The cysts are filled with serous fluid. This malformation may remain asymptomatic or cause different symptoms depending on the mass size or location $[5,6]$.

We present a case of a young girl routinely followed since birth for an antenatal diagnostic of retroperitoneal cystic lymphan-gioma with a favorable outcome towards regression but an evolving skin lymphangioma.

\section{Case Presentation}

This is the case of a seven-year-old girl, whose past medical history consists of a biamniotic bichorial twin pregnancy

*Corresponding author: Stessy Kutchukian, Department of Urology, Poitiers University Hospital, 2Rue de la Milétrie, 86021 Poitiers, France

Accepted: September 26, 2020

Published online: September 28, 2020

Citation: Kutchukian S, Potop D, Chalhoub K, et al. (2020) Retroperitoneal Cystic Lymphangioma: A Case Report of Spontaneous Regression and Review of Literature. Dermatol Arch 4(1):89-92 


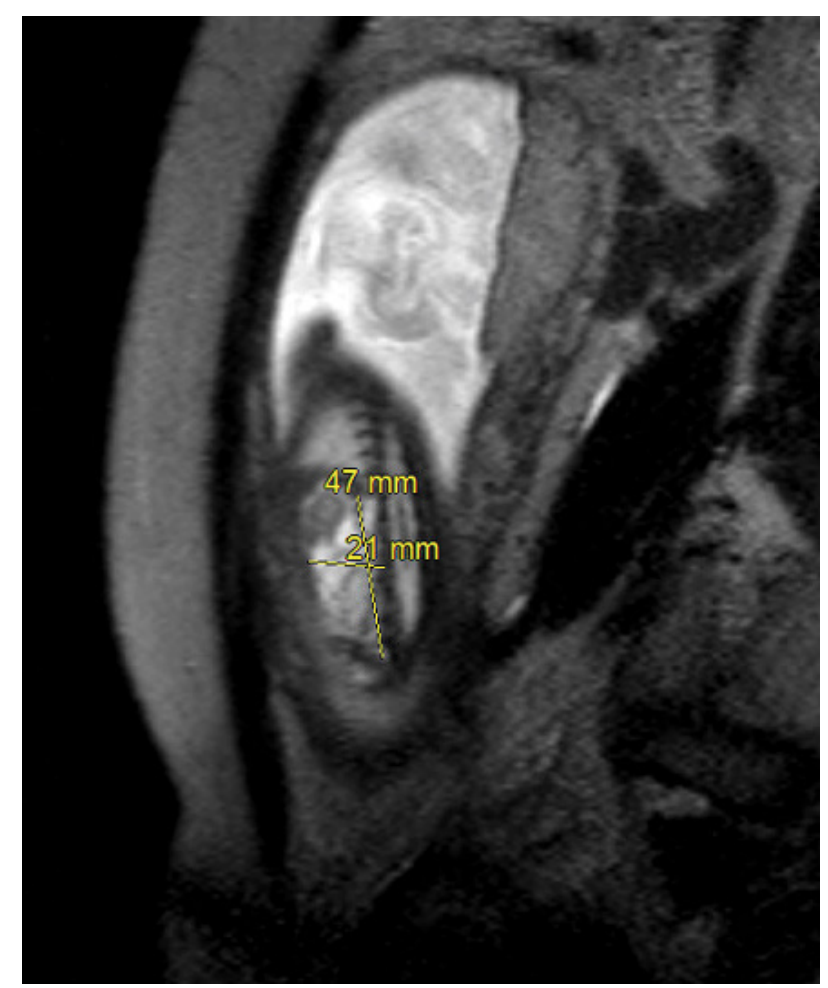

Figure 1: Fetal MRI (at 26 wGA) in T2 sequence with retroperitoneal $\mathrm{CL}$.

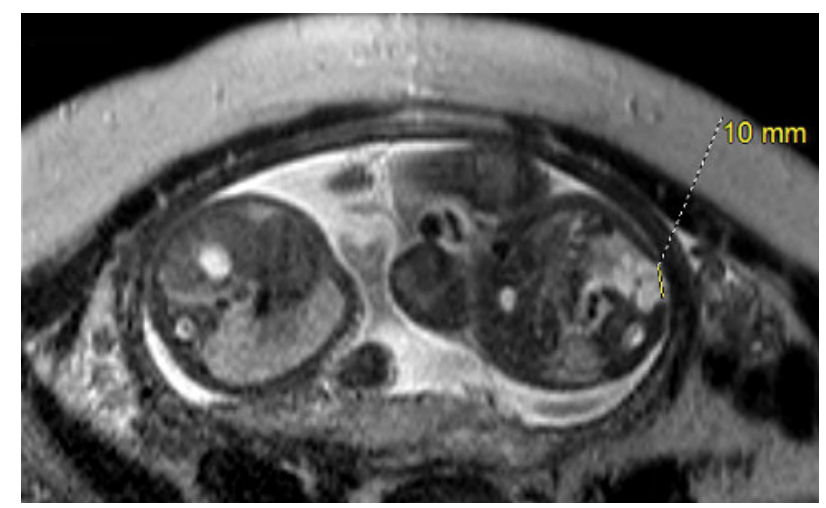

Figure 2: Fetal MRI (at 26 wGA) in T2 sequence with left lumbar subcutaneous extension.

in which one female fetus (fetus B) had a left retroperitoneal cystic malformation and the other male fetus (fetus $A$ ) was healthy. The mother was 29 years old an it was her first pregnancy with a body mass index (BMI) of 29.6 and blood type A + . The prena-tal ultrasound (US) at 19 weeks gestational age (wGA) showed a heterogeneous lesion in the left retro-renal lumbar region expanding as far as the left iliac fossa, with a subcutaneous extension towards the ipsilateral buttock. This lesion appeared isolated without any Doppler signal. The nuchal translucency of fetus B was normal. At 26 wGA a new morphological assessment was carried out with an ultrasound and a fetal MRI. The ultrasound showed the left retro renal lymphangioma with the same characteristics, without any increase in volume or com-pression. The MRI found a left retro-peritoneal mass of $47 \mathrm{~mm} \times 20 \mathrm{~mm} \times 30 \mathrm{~mm}$ diameter

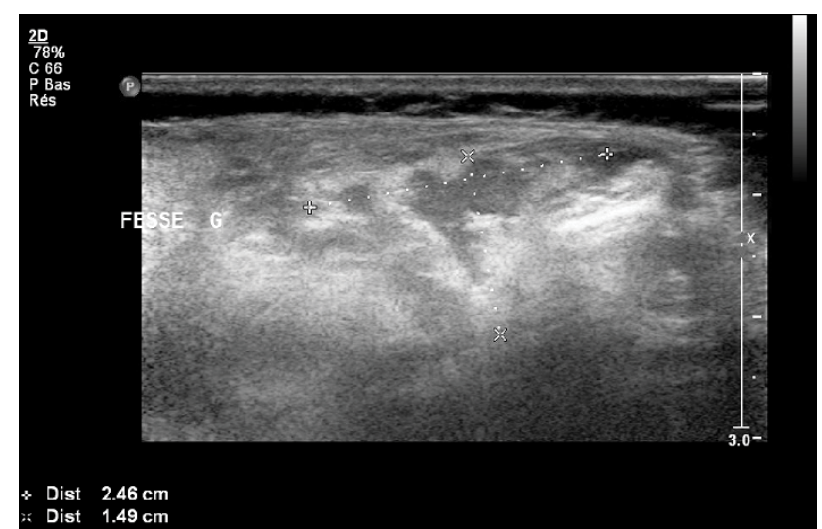

Figure 3: Post-natal ultrasound at age of 2 months.

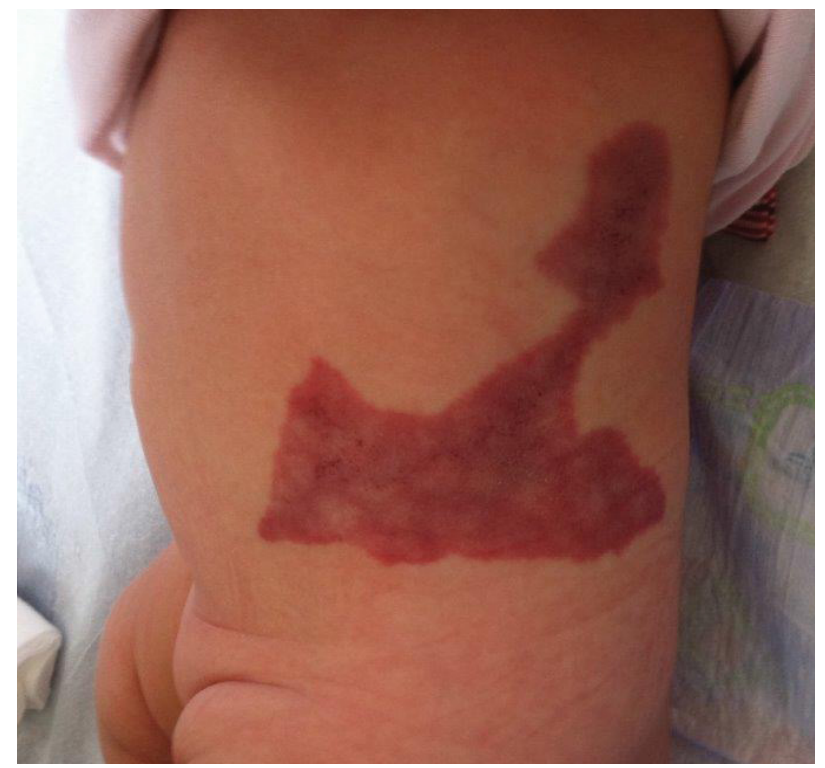

Figure 4: Lymphangioma on the skin on the left flank (1 year old).

posterior and medial to the left kidney, which stretches the left psoas and descends to the aortic bifurcation (Figure 1).

There was also a left lumbar subcutaneous extension of $10 \mathrm{~mm}$ (Figure 2).

No extension to the left buttock as seen on ultrasounds was noted. This mass was T1 hypointense and T2 fluid hypersignal with multiple partitions. The left kidney had a normal size and no other anomaly was identified. All the results were highly suggestive of CL. Being a competent regional Prenatal Diagnostic Center and having highly qualified pediatric radiologists, we considered that we have strong ar-guments to sustain this diagnostic. The twins were born naturally at 36 wGA and 3 days (foetus B: weight $2.025 \mathrm{~kg}$, height $44 \mathrm{~cm}$, cranial perimeter $32 \mathrm{~cm}$ ). Postnatal ultrasound showed a regression of the cystic lesions with signs of compression of adjacent organs and a normal renal parenchyma without dilation of the pelvicalyceal system. The cystic lesion was measured between 2.5 and $1.5 \mathrm{~cm}$ (Figure 3).

Upon clinical inspection, we noticed a lymphangioma measu-ring $8 \times 7 \mathrm{~cm}$ (Figure 4 ) on the skin on the left flank. 


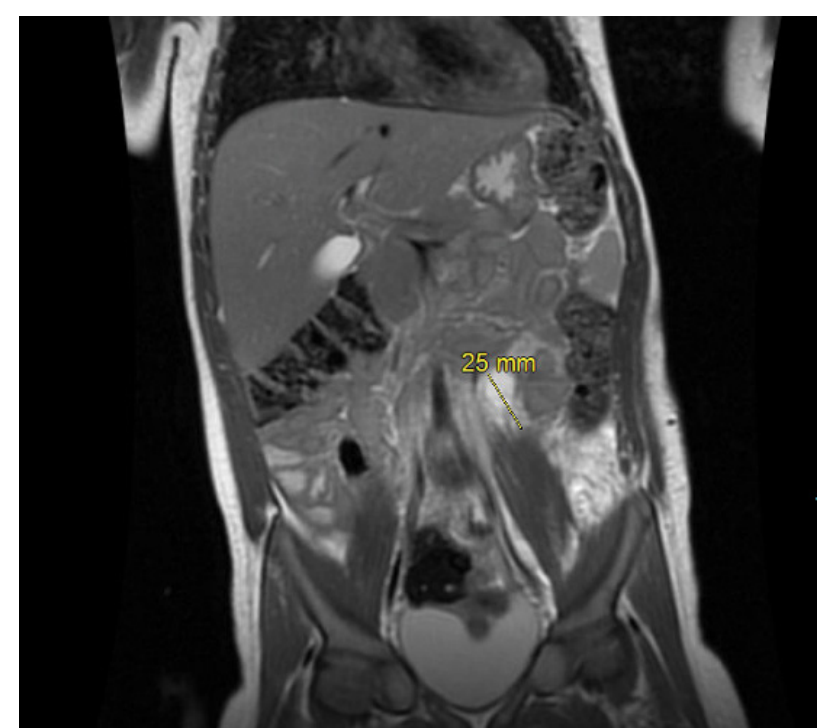

Figure 5: $\mathrm{MRI}$ in T2 sequence (at 2 years old).

Palpation yielded a fibrotic skin which was suspicious of subcutaneous lesion scarring.

The patient had a regular follow up, with clinical and ultrasound monitoring at birth, every 6 months for 3 years and then annually. At the age of 1 year, the retroperitoneal lesion remained completely asymptomatic. The child had a strictly normal examination of the abdomen. Even though in children the symptoms are difficult to asses, we relied on the eventual repercussions that the mass could have, but her renal function remained completely normal. On the other hand, a small purplish maculopapular rash appeared on the surface of the lymphangioma. An ultrasound evaluation showed an increasing lymphatic mal-formation of $40.3 \times 27.9 \times 12.2 \mathrm{~mm}$ against $24.6 \times 14.9 \mathrm{~mm}$. All in favor of a progressive lymphatic malformation associated with the appearance of lymphatic vesicles on the lymphangioma. At the age of 2 , the angioma measured $14 \mathrm{~cm}$ long and $11 \mathrm{~cm}$ wide. A new MRI was performed that found the retroperitoneal mass measuring $25 \mathrm{~mm} \times 25 \mathrm{~mm} \times$ $15 \mathrm{~mm}$ (Figure 5).

This lesion didn't seem to have an obvious connection with the cutaneous lesion, which associated a capillary component and dark papular bluish dilations, either of venous or lymphatic origin. Although the final diagnosis of cystic lymphangioma must be confirmed by pathology we decided not to take any risk by doing the biopsy given the regression of the lesion. During the follow up multiple hemorrhagic vesicles appeared on the surface of the malformation, being highly suggestive of a microcystic lymphangioma like a lymphangioma circumscriptum. Two laser tests were carried out on the angiomatous layer and on the lymphatic part respectively. The effect of these various tests were unsuccessful, leading to multiple surgical excisions at the age of 3 , then at the age of 4 and 6, before the recurrence of the lymphatic and capillary malformation of the left flank with hemorrhagic vesicles. The last resection was associated with pulsed dye laser on the remaining surface. Histological examination of the skin lesion confirmed a veno-lymphatic malformation, without any signs

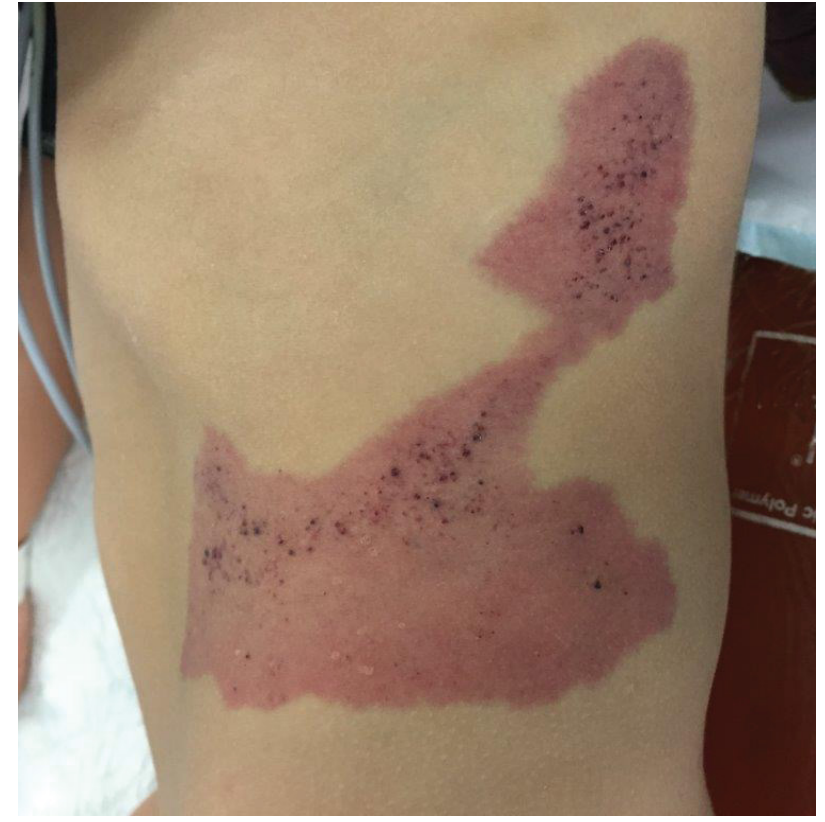

Figure 6: Cutaneous progression of the microcystic lym-phangioma with appearance of lymphatic vesicles (at 3 years old).

of malignancy. The samples showed capillary structures saturated with red blood cells in the papillary dermis. Deeper, we noted the presence of large veins and venules, which were associated with capillary hyperplasia in the adipocyte lobules. The blood-saturated vessels were irregularly arranged, plurifocal, sometimes with clus-ters lifting the epidermis, which appeared hyperplastic, without cytonuclear atypia and with a lack of expression of Glut-1 in immunohistochemistry. D2-40 was partially expressed showing that some vessels had lymphatic origins and CD31 expression was in favor of abnormal vessels. Follow-up was continued and we were facing a regression of the retroperitoneal lesion with a cutaneous progression of the veno-lymphatic malformation (Figure 6).

\section{Discussion and Review of Literature}

Abdominal cystic lymphangiomas are rare tumors with a repor-ted incidence between 1 in 20,000 - 250,000. Almost $90 \%$ are detected by the age of 2 , and most occur in the small bowel mesentery [7]. Lymphangiomas were first described by Koch in 1913 and their histogenesis is still uncertain. Two phenomenas can explain the development of this type of lesion. One is a congenital origin due to the lack of connections between a group of abdominal lymphatic channels and another is represented by the venous system, causing the isolation of a lymphatic bud which will develop into a cyst. The first suggests an acquired origin resulting from an anomalous development of lymphatic system, which involves the obstruction of lymphatic vessels due to inflammation, trauma or degeneration [8-10]. Three histological types of lymphangiomas are described: cystic, capillary and cavernous. Retroperitoneal lymphangioma is usually the cystic type. Although this lesion is benign, it can lead to significant morbidity due to its potential of growth and a strong tendency for secondary infection [11]. When the lesion is symptomatic, the clinical signs are re- 
lated to the large size or to a complication (rupture, infection, intracystic hemorrhage, compression or infiltration of vital structures). The malignant transformation is exceptional. The percentage of complications is rarely found in the literature if there is no treatment. However, in the case of microcystic lymphangiomas the most frequent complication is bleeding due to hemorrhagic vesicles. The diagnosis of $C L$ is suggested by imaging and it can be made in the prenatal period or later in life. On US CLs appear as a simple or multiloculated (honeycomb), anechoic, well defined, liquid tumors often containing thin septa. Subsequently MRI or Computed Tomographic (CT) scan define the structural details and assess the relationship with adjacent organs and the perivascular extension of the cystic lesion. Histologically, CLs are formed by cavities characterized by a thin irregular wall covered by endothelium resting on fibrous tissue containing lymphocytic islets and sometimes smooth muscle fibers and foam cells. In our case, the skin lesion is similar to a circumscriptum lymphangioma or superficial cutaneous lymphangioma. This skin malformation is rare, composed of translucent or hematic ve-sicles. There are different types of treatment, but often with multiple recurrences. Pulsed dye laser with surgery may be an effective treatment option for hematic vesicles $[12,13]$. The surgical treatment, if performed, must be complete to avoid recurrence. In our case it was necessary to operate only the symptomatic skin lesion of the left flank.

\section{Conclusion}

Retroperitoneal $\mathrm{CL}$ is a benign tumor of the lymphatic system. The clinical symptomatology is polymorphic and nonspecific. The diagnosis suggested by imaging can be made during the prenatal period. Regular clinical and imaging follow-up may appear to be a therapeutic strategy in lesions that seem to be heading towards complete regression.

\section{Author Disclosure Statements}

Nothing to disclose.

\section{References}

1. M Tripathi, S Parshad, RK Karwasra, et al. (2015) Retroperito-neal lymphangioma in an adult: A case report of a rare clinical entity. Case Rep Surg.

2. BJW Chew, H Dagash, MM Khare (2019) Intra-abdo-minal cystic lymphangioma. The Journal of Pediatrics 205: 288-288.e1.

3. J Xiao, Y Shao, S Zhu, et al. (2020) Characteristics of adult abdominal cystic lymphangioma: A single-center Chinese cohort of 12 cases. BMC Gastroenterol 20: 244.

4. E Târcoveanu, R Moldovanu, C Bradea, et al. (2016) Laparoscopic treatment of in-traabdominal cystic lymphangioma. Chirurgia (Bucur) 111: 236-241.

5. S Godart (1966) Embryological significance of Iymphangioma. Arch Dis Child 41: 204-206.

6. P Ravasse, M Le Treust, C Levesque, et al. (1995) Le lymphan-giome kystique rétropéritonéal: Une tumeur d'expression clinique très polymorphe. À propos de trois cas. Archives de Pédiatrie 2: 232-236.

7. Y Hamaguchi, S Arita, N Sugimoto, et al. (2020) Laparoscopic resection of abdominal cystic lymphangioma derived from lesser omentum. Medicine (Baltimore) 99.

8. K Koch (1913) Beiträge zur Pathologie der Bauchspeichel-drüse. Virchows Arch path Anat 214: 180-206.

9. I Roisman, J Manny, S Fields, et al. (1989) Intra-abdominal lymphangioma. Br J Surg 76: 485-489.

10. JA Perkins, SC Manning, RM Tempero, et al. (2010) Lym-phatic malformations: Current cellular and clinical investi-gations. Otolaryngol Head Neck Surg 142: 789-794.

11. M Gachabayov, K Kubachev, E Abdullaev, et al. (2016) A huge cystic retroperitoneal lymphangioma presenting with back pain. Case Rep Med.

12. M Heller, S Mengden (2008) Lymphangioma circumscrip-tum. Dermatol Online J 14: 27.

13. CH Lai, SG Hanson, SB Mallory (2001) Lymphangioma circumscriptum treated with pulsed dye laser. Pediatr Dermatol 18: 509-510.

DOI: $10.36959 / 661 / 309$ 